\title{
Human umbilical perivascular cells (HUCPVCs): a novel source of mesenchymal stromal-like (MSC) cells to support the regeneration of the testicular niche
}

\author{
Leila Maghen ${ }^{1, *}$, Ekaterina Shlush ${ }^{1, *}$, Itai Gat ${ }^{1,5}$, Melissa Filice ${ }^{1}$, Tanya A Barretto ${ }^{1}$, Keith Jarvi ${ }^{2}$, \\ Kirk Lo ${ }^{2}$, Andrée Gauthier-Fisher ${ }^{1}$ and Clifford L Librach ${ }^{1,3,4}$ \\ ${ }^{1}$ CReATe Fertility Centre, Toronto, Ontario, Canada, ${ }^{2}$ Division of Urology, Mount Sinai Hospital, Toronto, Ontario, \\ Canada, ${ }^{3}$ Department of Obstetrics \& Gynecology, University of Toronto, Toronto, Ontario, Canada, \\ ${ }^{4}$ Department of Gynecology, Women's College Hospital, Toronto, Ontario, Canada, and ${ }^{5}$ Pinchas Borenstein Talpiot \\ Medical Leadership Program, Sheba Medical Center, Tel HaShomer, Ramat Gan, Israel, affiliated with the Sackler \\ School of Medicine, Tel-Aviv University, Tel-Aviv, Israel
}

Correspondence should be addressed to A Gauthier-Fisher or C Librach; Email: andree@createivf.com or drlibrach@createivf.com

*(L Maghen and E Shlush contributed equally to this work $)$

\begin{abstract}
The expansion of functional testicular biopsy-derived human spermatogonial stem cells (hSSC) ex vivo may enable the restoration of fertility in pre-pubertal males having undergone gonadotoxic therapies or men with severe male factor infertility. Various somatic cells are known to regulate SSC homeostasis and spermatogenesis in the developing and adult testis. Prior attempts to recapitulate this niche demonstrated the requirement of feeder cells, such as endogenous testicular somatic cells, for germ cell expansion ex vivo. However, this strategy has limitations in the expansion of hSSCs from tissue biopsies for which spermatogenesis is absent or defective. Our aim was to evaluate the first trimester human umbilical cord perivascular cells (FTM HUCPVCs), a novel source of mesenchymal stromal-like cells (MSCs), as potential human feeder cells for standardized hSSC expansion ex vivo. Targeted RNA sequencing analysis demonstrated that CD90+ve FTM HUCPVCs expanded in vitro under germ cell culture conditions express a profile of targeted testicular-associated transcripts that is similar to cultured human CD90+ve testicular adherent cells (hTACs) and secrete LIF, FGF2 and BMP4, key growth factors known to regulate spermatogenesis. We also demonstrated that mitotically inactivated FTM HUCPVCs support the expansion of mouse germ cells and putative SSCs ex vivo and that FTM HUCPVC transplantation promotes in vivo germ cell regeneration after mono-2-ethylhexyl phthalate (MEHP)-induced seminiferous tubule damage in a murine model, including a partial reconstitution of tubular cellular architecture and reestablishment of DAZL and acrosin+ve germ cell layers. Together, these data suggest that FTM HUCPVCs have phenotypical and functional properties that may support repair of the human testicular niche. Reproduction (2017) 153 85-95
\end{abstract}

\section{Introduction}

The development of methods to propagate human spermatogonial stem cells (hSSCs) ex vivo may be required steps for fertility preservation and restoration strategies for childhood cancer survivors with cryopreserved testicular tissue. This strategy may also have potential for use in treatment strategies for infertile men with severely defective spermatogenesis, such as those with non-obstructive azoospermia (NOA). The development of clinically compatible cell culture methods, including the removal of animal-derived products from such protocols, is a key step. In addition, the development of ex vivo culture models may provide a valuable research tool to improve our understanding of normal human spermatogenesis and some cases of male infertility. Multiple somatic cell types in the testis, including seminiferous tubule Sertoli cells and interstitial cells that include Leydig cells, peritubular myoid cells and vascular cell types, create a microenvironment that, together with germ cell feedback mechanisms, orchestrates spermatogonial stem cell (SSC) maintenance, self-renewal, symmetric and asymmetric mitotic divisions as well as meiotic differentiation of germ cells (Oatley \& Brinster 2012). Feeder and feederfree culture systems supplemented with testicular nicherelevant factors have enabled the functional propagation of murine SSCs that could generate healthy neonates after transplantation (Kanatsu-Shinohara \& Shinohara 2010, Choi et al. 2014). However, animal componentfree culture conditions are obligatory for human SSC expansion.

Previous studies have demonstrated that endogenous somatic cells that adhere and proliferate with germ cells 
in culture can support the expansion of germ cells with SSC properties in 'feeder-free' cultures (Sadri-Ardekani et al. 2009, 2011). However, the expansion of human spermatogonia in culture has had limited inter-laboratory reproducibility. This could be due to technical variation between protocols, differing ability to control the overproliferation of endogenous somatic cells that inhibit germ cell expansion or the potential heterogeneity of available research samples. With the exception of a recent study by Smith and coworkers, tissue samples in these studies have largely consisted of orchiectomies for testicular cancer or pain, and microscopic testicular sperm extraction (microTESE) samples from men with azoospermia. More recently, a CD90+ve subpopulation of human testicular adherent somatic cells (hTACs) with mesenchymal properties was selectively expanded in hSSC culture conditions, gamma irradiated and then used as a feeder cell to support the expansion and passaging of putative SSEA4+ve SSCs for up to 3 weeks (Smith et al. 2014). We hypothesize that an alternate source of MSC mimicking properties of the testicular mesenchymal stromal cells described by Smith and coworkers could provide an optimal and standardized cell feeder system for the long-term xeno-free expansion of hSSCs, especially from tissue samples with absent or abnormal spermatogenesis.

The perivascular region of the first trimester (FTM) and term human umbilical cord contains a rich source of mesenchymal stromal cells with pericyte properties (HUCPVCs) that can be expanded in vitro (Sarugaser et al. 2005, Hong et al. 2013). We have shown that the younger FTM HUCPVCs appear to have a greater capacity for multi-potential differentiation, greater immunoprivilege properties and enhanced paracrine effects compared with their term counterparts (Hong et al. 2013, Szaraz et al. 2016, E Shlush, L Maghen, S Swanson, S Kenigsberg, S Moskovtsev, T A Barretto, A Gauthier-Fisher and C L Librach, unpublished observations). We have also demonstrated that these cells express and secrete factors that are known to be important regulators of multiple testicular cell lineages, including FGF2, GDNF, LIF and BMP4 (Shlush et al. unpublished observations). Here, we explored the use of FTM HUCPVCs as a potential replacement for requisite hTACs during germ cell expansion and/or differentiation. Specifically, the objectives of this study were to evaluate the functional supportive properties of FTM HUCPVCs with regard to germ cell expansion in vitro, as well as regeneration in vivo.

\section{Materials and methods}

\section{FTM HUCPVC and hTAC isolation and culture}

Previously established lines of FTM HUCPVCs $(n=3$, normal XY karyotype; \#REB No. 454-2011, Sunnybrook Research Institute; REB \#28889, University of Toronto, Canada) were expanded in aMEM (ThermoFisher Scientific) $+10 \%$ FBS (GE Health Care Life Sciences; Lot \# AWK24007), as described previously (Hong et al. 2013). Adult hTACs were obtained with written informed consent from a portion of orchiectomy specimens and microTESEs (University of Toronto REB \# 30252 and REB \#14-0032-E Mount Sinai Hospital, Toronto, Canada). Upon collection, tissue was transferred to human tubal fluid (HTF) HEPES (In Vitro Care, Frederick, MD, USA) containing $20 \%$ FBS and 1\% ABAM (GE Health Care Life Sciences) and processed at the CReATe Fertility Centre (Toronto, Canada). In some cases, testicular tissue was cryopreserved prior to cell isolation procedures. For cryopreservation, human testicular tissue was washed three times in PBS and was cut into $2-4 \mathrm{~mm}^{3}$ pieces in DMEM/F12 (ThermoFisher Scientific) $+20 \%$ FBS. Twenty to thirty $2-4 \mathrm{~mm}^{3}$ tissue pieces were placed in $1.5 \mathrm{~mL}$ cooled cryovial containing cryoprotectant medium, consisting of DMEM-F12, 20\% FBS (GE Health Care Life Sciences) and 5\% DMSO (Sigma-Aldrich). The tissue samples were transferred into liquid nitrogen after overnight incubation at $-80^{\circ} \mathrm{C}$. For fresh and thawed tissue processing, the tissue was cut into $2-4 \mathrm{~mm}^{3}$ pieces and the tubules were teased apart with forceps in DMEM/F12 containing non-essential amino acids (ThermoFisher Scientific), $4 \mathrm{mM}$ L-glutamine (ThermoFisher Scientific), sodium bicarbonate 7.5\% (Sigma-Aldrich), $40 \mu \mathrm{g} /$ $\mathrm{mL}$ gentamicin (ThermoFisher Scientific), penicillin (100IU/ $\mathrm{mL})$ and streptomycin $(100 \mu \mathrm{g} / \mathrm{mL}$, ThermoFisher Scientific). Two enzymatic digestion steps were performed with $1 \mathrm{mg} / \mathrm{mL}$ collagenase type I (Sigma-Aldrich; Cat. LS004196), $1 \mathrm{mg} / \mathrm{mL}$ hyaluronidase type II (Sigma-Aldrich; Cat. H2126) and $1 \mathrm{mg} /$ $\mathrm{mL}$ trypsin (Sigma-Aldrich; Cat. T1005) for $15 \mathrm{~min}$ at $32^{\circ} \mathrm{C}$ with gentle shaking followed by $1 \mathrm{mg} / \mathrm{mL}$ collagenase type I and $1 \mathrm{mg} / \mathrm{mL}$ hyaluronidase for $30 \mathrm{~min}$ at $32^{\circ} \mathrm{C}$ with gentle shaking (He et al. 2012).

Eight mg/mL DNasel (Sigma-Aldrich; Cat. DN25-100MG) was used in all steps of isolation. To separate the somatic cells from germ cells, dissociated testicular cells were cultured overnight on $0.2 \%$ gelatin-coated plate $\left(30-50 \mathrm{~K}\right.$ cells $\left./ \mathrm{cm}^{2}\right)$ in DMEM/F12 containing $20 \% \mathrm{FBS}$ at $37^{\circ} \mathrm{C}, 5 \% \mathrm{CO}_{2}$. FTM HUCPVCs and somatic cells (after differential plating) were both cultured on laminin-coated plates $(20 \mu \mathrm{g} / \mathrm{mL}, \quad B D$ Biosciences, Mississauga, ON, Canada; Cat. 354232) in supplemented StemPro-34 media (ThermoFisher Scientific Cat. 10639-011) +2\% FBS (GE Health Care Life Sciences) (Kanatsu-Shinohara et al. 2003). The following growth factors and reagents were added to the media: GDNF $\left(10 \mathrm{ng} / \mathrm{mL}, \mathrm{R} \& D\right.$ system, Cat212-GD-010), LIF $\left(10^{3} \mathrm{U} / \mathrm{mL}\right.$, EMD Millipore; Cat. GF342), EGF $(20 \mathrm{ng} / \mathrm{mL}$, Peprotech, Rocky Hill, NJ, USA; Cat. AF-100-15) and $\beta F G F$ (10 ng/mL, Peprotech; Cat. Af-100-18B). In some experiments, parallel FTM HUCPVC cultures were gamma irradiated with Cesium-137 for $13.5 \mathrm{~min}$ at $150 \mathrm{rad} / \mathrm{min}$ (Smith et al. 2014) at the University of Health Network's Animal Resource Centre facility (Toronto, Canada).

\section{Primary mouse spermatogonial cell cultures}

All work was performed in accordance with CACC guidelines and with Toronto Centre for Phenogenomics animal facility approval (Animal User Protocol \#0245). 
Mouse spermatogonial stem cell cultures were prepared as described previously (Kanatsu-Shinohara et al. 2003), with minor modifications. Postnatal day 4-7 CD-1 mouse male (Crl:CD1(ICR), TCP stocks) were decapitated with scissors. Testes were isolated on ice, separated from the tunica and digested in $1 \mathrm{mg} / \mathrm{mL}$ collagenase solution (Sigma-Aldrich) for $15 \mathrm{~min}$ at $37^{\circ} \mathrm{C}$ with gentle shaking. The cell pellet was washed with HBSS and further digested in $0.25 \%$ trypsin (ThermoFisher Scientific) containing $7 \mathrm{mg} / \mathrm{mL}$ DNAse I (SigmaAldrich) for $10 \mathrm{~min}$ at $37^{\circ} \mathrm{C}$, with regular shaking to dissociate the seminiferous tubules. The cells were resuspended in StemPro34 $+2 \%$ StemPro Supplements (ThermoFisher Scientific) and dissociated manually by trituration using a $1 \mathrm{~mL}$ pipette tip and isolated by centrifugation at $200 \mathrm{~g}$. The cell pellet was resuspended in complete medium, as previously described (Kanatsu-Shinohara et al. 2003), filtered through a $70 \mu \mathrm{m}$ cell strainer (ThermoFisher Scientific) and viable cells obtained were counted using the Countess Automated Cell Counter (ThermoFisher Scientific). To separate somatic cells from germ cells, $2.5 \times 10^{6}$ (freshly dissociated cells) were plated on $0.2 \%$ gelatin-coated culture dishes and incubated at $34^{\circ} \mathrm{C}$ overnight. Floating cells $\left(5 \times 10^{4} / \mathrm{cm}^{2}\right)$ were then plated on mouse embryonic fibroblast feeders (MEF) (Applied Stem Cell, Milpitas, CA, USA; Lot \#F1201241612) or FTM HUCPVC feeders. Floating cells were plated on respective feeders at $7.8 \times 10^{3}$ cells $/ \mathrm{cm}^{2}$. MEF feeders were prepared according to manufacturer instructions and FTM HUCPVC feeders were prepared in a similar manner. Briefly, cryostocks of MEFs were thawed and cultured in ESC-Sure DMEM (Applied Stem Cell; Cat. ASM-5001) containing 10\% FBS (GE Health Care Life Sciences), 0.1 mM MEM Non-essential amino acids (ThermoFisher Scientific), $1 \mathrm{mM}$ Sodium Pyruvate and $1 \times$ GlutaMAX (ThermoFisher Scientific). Previously established lines of FTM HUCPVCs ( $n=2$, passage $3-5)$ were expanded in standard culture conditions ( $\alpha M E M+10 \%$ FBS) for at least 2 passages. $70 \%$ confluent culture dishes were incubated for $5 \mathrm{~h}$ with $15 \mu \mathrm{g} / \mathrm{mL}$ mitomycin C (Sigma-Aldrich), rinsed with PBS and passaged using TrypLE (ThermoFisher Scientific). Feeder cells $\left(7500 \mathrm{cells} / \mathrm{cm}^{2}\right)$ were plated on gelatin-coated 12- or 6-well tissue culture dishes (BD Biosciences) and used to co-culture germ cells within 24-72 h. Complete media was changed every 4 days.

\section{Immunocytochemistry and microscopy}

Cells were washed with PBS and fixed in 4\% paraformaldehyde for $20 \mathrm{~min}$. After permeabilization with $0.1 \%$ Triton-X (SigmaAldrich) in PBS, cells were blocked with 5\% normal goat serum (Jackson Immunoresearch Laboratories) in 1\% BSA (ThermoFisher Scientific)-PBS for $1 \mathrm{~h}$ at room temperature and incubated at $4{ }^{\circ} \mathrm{C}$ overnight with the following primary antibodies: rabbit anti-human CD90/(a.k.a Thy1) antibody (1:100, Abcam; Cat. Ab133350), anti-CD44 (BD Biosciences; Cat \#559942) and rabbit anti-Sox9 (1:250, Abcam; Cat $\mathrm{Ab34139).} \mathrm{For} \mathrm{BrdU} \mathrm{staining} \mathrm{of} \mathrm{mouse} \mathrm{spermatogonial}$ stem cells and feeder co-cultures, $10 \mu \mathrm{mol} / \mathrm{mL}$ BrdU (BD Biosciences; Cat. 552598) was added to the cells and incubated for $72 \mathrm{~h}$ at $37^{\circ} \mathrm{C}$. Cells were washed with cold HBSS (ThermoFisher Scientific; Cat, 14175-095), fixed with $70 \%$ ethanol and treated with $2 \mathrm{~N} \mathrm{HCl}$ for $10 \mathrm{~min}$, and $0.1 \mathrm{mM} \mathrm{Na}{ }_{2} \mathrm{~B}_{4} \mathrm{O}_{7}, \mathrm{pH} 8.5$ for $10 \mathrm{~min}$. Cells were washed with PBS before overnight incubation with primary rabbit anti-DAZL antibody (1:400, Abcam; Cat. AB34139), antiGPR125 (1:200, Abcam; Cat. AB51705) and mouse anti-BrdU $(6 \mu \mathrm{g} / \mathrm{mL}$ EMD Millipore; Cat. MAB3424). The cells underwent three 5 -min washes with $1 \%$ BSA-PBS and were incubated at room temperature for $1 \mathrm{~h}$ with secondary antibodies; Alexa 488 Goat anti-mouse and Alexa 555 Goat anti-Rabbit (1:500, Invitrogen Life Technologies). Nuclei were counterstained with Hoechst (1:2000, ThermoFisher Scientific) for 3 min followed by three 5 -min washes with $1 \%$ BSA-PBS. Negative controls in which primary antibodies were omitted were also included. Slides were protected with PermaFluor Mounting Media (ThermoFisher Scientific). Fluorescence images were observed and captured using the EVOS fluorescence microscope (ThermoFisher Scientific).

\section{Fluorescence-activated cell sorting (FACS)}

Adherent cells were trypsinized using TrypLE (Invitrogen Life Technologies). Single cells were incubated with antihuman PE-conjugated CD90 (1:40, BD Biosciences; Cat. 555596) for $30 \mathrm{~min}$ at $4^{\circ} \mathrm{C}$, after washing with $3 \%$ FBS-PBS. Propidium iodide (PI) (Sigma-Aldrich) was used to determine the cell viability. The CD90+ve subpopulations of FTM human umbilical cord perivascular cells $(n=4)$ and human adult testicular somatic cells (expanded in culture, $n=8$ ) were sorted directly into RNA lysis buffer (Norgen Biotek Corporation, Burlington, ON, Canada) using the FACS Ariall-SC BRV (BD Biosciences) at the Hospital for Sick Children-UHN flow cytometry facility (Toronto, Ontario, Canada). The frequency of CD90+ve cells was determined using FACS DIVA (BD Biosciences).

\section{Targeted RNA sequencing}

RNA samples were prepared using the RNA/DNA Purification Micro Kit (Cat. 50300, Norgen Biotek Corporation) according to the manufacturer's instructions. RNA quantification and quality assessments were performed using the Bioanalyzer 2100 (Agilent Genomics) at the Princess Margaret Genomic Centre (Toronto, Canada). RNAseq libraries were prepared with Ion Ampliseq RNA library kit (Invitrogen Life Technologies). Briefly, $10 \mathrm{ng}$ of RNA were reverse transcribed into cDNA using a targeted RNA sequencing custom panel including 87 amplicons outlined in Supplementary Table 1, see section on supplementary data given at the end of this article (Invitrogen Life Technologies) according to the manufacturer's instructions. Primer sequences were digested and barcode-adaptors were ligated to cDNAs followed by purification and amplification steps. Purified, amplified libraries were quantified using chip-based capillary electrophoresis (Bioanalyzer 2100, Agilent Genomics). Ion 316 Chip Kit v2 chips (Invitrogen Life Technologies) were used for sequencing. Up to 16 samples were loaded per chip using $30-50$ pmol of the prepared library. Next-generation sequencing (NGS) was performed using the Ion PGM sequencer (Invitrogen Life Technologies). BAM files of aligned data were uploaded from lon Reporter 
(Invitrogen Life Technologies) to Partek Flow (Partek, St Louis, $\mathrm{MO}$, USA) for bioinformatics analysis. Aligned files were subjected to post-alignment QC to determine the number of usable reads for each sample, degree of alignment and quality score. Samples with greater than 65,000 and up to 300,000 total reads showing greater than $98 \%$ alignment were included in the analysis. Targeted RNAseq analysis was performed by quantifying aligned reads to transcriptome using the Partek/ EM platform and Homo sapiens (human) - hg19 canonical transcripts as the genome build and the custom testicular amplicon panel as the annotation model. Quantification was performed with strict paired-end compatibility and minimal read overlap, with the feature option set at $80 \%$ of read length. Differential gene expression and statistical analysis was performed using Partek Flow (Partek) by comparing hTAC vs FTM HUCPVC transcript reads normalized to the total number of reads for each sample. A PCA map was generated using Partek Flow (Partek), and GraphPad Prism (GraphPad Software) was used to plot mean \pm S.D. normalized reads for each amplicon on the panel.

\section{Collection and analysis of conditioned media by ELISA}

Overall, 400,000 HUCPVCs plated in a $10 \mathrm{~cm}^{2}$ dish (BD Biosciences) were cultured in Stempro-34 media (ThermoFisher Scientific) (basal media) supplemented with $2 \%$ FBS for $24 \mathrm{~h}$ until they reached $70 \%$ confluency, at which point they were rinsed twice with PBS and incubated in unsupplemented StemPro-34 media for $1 \mathrm{~h}$. Unsupplemented basal media was replaced, and cells were incubated for $72 \mathrm{~h}$. Media was collected, centrifuged at $3000 \mathrm{rpm}$ for $10 \mathrm{~min}$ and snap frozen in $1-5 \mathrm{~mL}$ aliquots. Cells were harvested and counted using the Countess Automated Cell Counter (Invitrogen Life Technologies). For ELISA analysis, conditioned media was thawed and concentrated using $150 \mathrm{~K}$ MWCO protein concentrators (Pierce Brand, ThermoFisher Scientific; Cat. P187748). Basal media alone was used as a control. ELISA analysis for human BMP4, LIF, bFGF and GDNF was performed according to manufacturer instructions, including the kit standards (RayBiotech, Norcross, GA, USA), and analyzed in duplicates on a Multi-Mode Microplate Reader F5 (Molecular Devices, Sunnyvale, CA, USA). The blank OD value measured for all experiments was subtracted from basal media, FTM HUCPVC and hTAC conditioned media OD values. The quantity of each factor was calculated using the standard curve, dilution factor and final volume collected and was expressed as the total amount of each factor secreted from the originally plated 100,000 cells.

\section{MEHP-induced testicular injury and histological analysis}

In accordance with CACC guidelines and with approval from the Toronto Centre for Phenogenomics animal facility (Toronto, Canada) (AUP \#16-228H), 6-week-old male NOD SCID

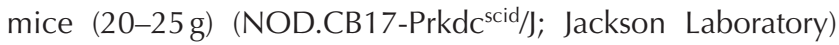
fasted overnight were gavaged with $1 \mathrm{~g} / \mathrm{kg}$ mono-2-ethylhexyl phthalate (MEHP; Accustandard, New Haven, CT, USA) dissolved in corn oil. Controls (no injury, $n=2$ ) were gavaged with corn oil. Undifferentiated FTM HUCPVCs were labeled by incubating them with $0.4 \mu \mathrm{L}$ PKH26 Red Fluorescent in $100 \mu \mathrm{L}$ diluent $\mathrm{C} / 1 \times 10^{6}$ cells, as per manufacturer's instructions (Sigma-Aldrich). Twenty-four hours after MEHP treatment, intra-testicular injection of saline (control, $n=2$ ) or 50,000 undifferentiated PKH-26-labled FTM HUCPVCs $(n=5)$ in a final volume of $20 \mu \mathrm{L}$ was performed in a biological safety cabinet on animals anesthetized with isoflurane (2-4\%, Sigma-Aldrich) by performing a small lower abdominal incision. Animals were monitored daily and given meloxicam ( $2 \mathrm{mg} / \mathrm{kg}$; Boehringer-Ingelheim, Ingelheim, Germany) for 2 days following this procedure. Animals were killed by $\mathrm{CO}_{2}$ asphyxiation at 1-4 weeks after MEHP treatment. The testes were dissected and (1) transferred to Tissue-TekROCT (Sakura Finetek USA Inc, Torrance, CA, USA) and processed for frozen sections or (2) formalin-fixed and processed for paraffin embedding and $\mathrm{H} \& \mathrm{E}$ staining at The Centre for Modeling of Human Disease, Pathology Core, Mount Sinai Hospital (Toronto, Canada). Images were captured using at $40 \times$ bright field using The Hamamatsu Whole Tissue Slide scanner (Olympus) and analyzed using NanoZommer 2.0 RS software (Hamamatsu, NJ, USA). The degree of restoration of the seminiferous tubule germ cell layer architecture was determined by blinded assessment of over 300 tubules per animal covering 3 testis sections that each represented a separate third of the tissue for each animal. Mean per treatment group is reported, together with standard error of the mean. Statistical analysis was performed using ANOVA followed by Tukey's multiple comparisons test and differences between groups were considered significant when $P<0.05$. The reports are compliant with ARRIVE guidelines for reporting in vivo experiments.

\section{Immunohistochemistry}

Sections were deparaffinized and rehydrated using xylene and ethanol respectively. Epitope retrieval was performed using $10 \mathrm{mM}$ sodium citrate (Sigma-Aldrich) at $95-100^{\circ} \mathrm{C}$ for 20 min. To block the endogenous peroxidase activity of the tissue, sections were incubated with $3 \% \mathrm{H}_{2} \mathrm{O}_{2}$ solution for 5 min. Sections were blocked for $1 \mathrm{~h}$ with $10 \% \mathrm{BSA}+0.3 \%$ Triton-X in PBS at room temperature and incubated at $4{ }^{\circ} \mathrm{C}$ overnight with the primary antibodies; anti-Acrosin (1:5, Santa Cruz Biotechnology; Cat. sc-46284), DAZL (1:50, Abcam; Cat. Ab34139). The sections were washed in PBS, 3 times for $10 \mathrm{~min}$ each, and then were incubated at room temperature for $1 \mathrm{~h}$ with secondary antibodies; Alexa 488 Goat anti-rabbit or donkey anti-goat (both 1:500, Invitrogen Life Technologies). Nuclei were counterstained with Hoechst (1:2000; Invitrogen Life Technologies) for 2 min followed by three 5-min washes with PBS. Slides were protected with PermaFluor mounting media (ThermoFisher Scientific) and then were scanned with a Pannoramic 250 Flash II slide scanner (3DHistech, Budapest, Hungary) at the imaging facility at the Hospital for Sick Children (Toronto, Ontario).

\section{Data analysis}

All results were generated from at least three independent experiments using 2-3 independent FTM HUCPVC lines 
or testicular tissue samples. The results were presented as mean \pm S.E.M. unless otherwise indicated. GraphPad Prism Software (GraphPad Software) was used for statistical analysis. Statistical significance was determined using Student's $t$ test or, when more than 2 conditions were tested, using ANOVA followed by Tukey's multiple comparisons test, and differences were considered significant when $P<0.05$.

\section{Results}

\section{FTM HUCPVCs share molecular properties with human adult testicular adherent/somatic cells}

We compared the morphological and molecular properties of FTM HUCPVCs with those of established cultures of human testicular adherent cells (hTACs) expanded in culture conditions previously used for the expansion of hSSCs (Sadri-Ardekani et al. 2011). When transferred onto laminin-coated plates and expanded in supplemented StemPro34, both hTACs and FTM HUCPVCs displayed fibroblast-like bipolar morphologies (Fig. 1A). Immunocytochemical analysis revealed that the majority of both cell types co-express CD44 and CD90, as well as CD44 and Sox9 (Fig. 1B), markers previously associated with HUCPVCs and MSCs (Dominici et al. 2006, Hong et al. 2013) and also showed to be expressed in FTM HUCPVCs expanded in regular MSC conditions (Fig. 1B, bottom panels). Flow cytometric analysis of CD90 expression in primary and passage 2 hTACs revealed that a CD90+ve subpopulation of adherent cells are selectively expanded during the culture of hTACs in SSC conditions $(46.7 \pm 2.5 \%$ vs $96.1 \pm 0.7 \%)$. This is not significantly different from the proportion of CD90+ve cells in FTM HUCPVC cultures under the same culture conditions $(83.0 \pm 6.8 \%, P>0.05)$ (Fig. 1D and E). A focused gene expression profile of 87 testicular lineage-associated transcripts (as outlined in Supplementary Table 1) was compared between CD90+ve cells isolated by FACS from expanded hTACs and from FTM HUCPVCs (2 passages in supplemented StemPro34-based conditions). Both hTACs and FTM HUCPVCs tended to express high levels of genes associated with somatic cells and MSCs and low levels of genes associated with germ cell, Leydig cells or Sertoli cells (Fig. 2A). A principle components analysis revealed that CD90+ve hTACs (passage 2) clustered very closely with FTM HUCPVCs (Fig. 2B). A detailed analysis of transcripts associated with MSC lineages showed that, overall, hTACs and FTM HUCPVCs expressed similar levels of MSC lineage markers including THY1 (CD90), NT5E (CD73), CD44 and SOX9 (Fig. 2C), consistent with the ICC data mentioned previously.

Out of the 87 transcripts on the panel, 11 (13\%) were found to be significantly upregulated in expanded hTACs when compared to FTM HUCPVCs, and 8 $(9 \%)$ were found to be significantly downregulated $(P<0.05)$. Forty-five $(52 \%)$ transcripts were found to be highly expressed (>200 normalized reads) by both cell types. Of these markers, 7 were found to be upregulated more than 2-fold in expanded hTACs when compared to FTM HUCPVCs. These included the testicular somatic cell-associated markers ENG, STAR and GATA4; extracellular matrix molecule LAMB1; as well as paternally imprinted gene $\mathrm{H} 19$; pluripotencyassociated KLF4 and cell cycle-associated MAP3K12
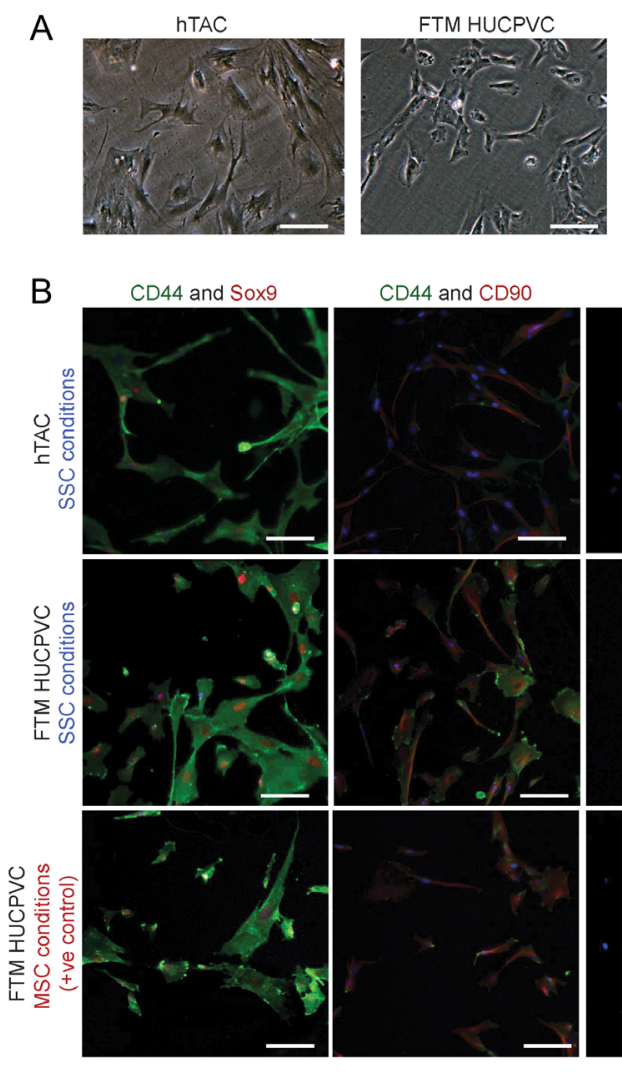

Negative control
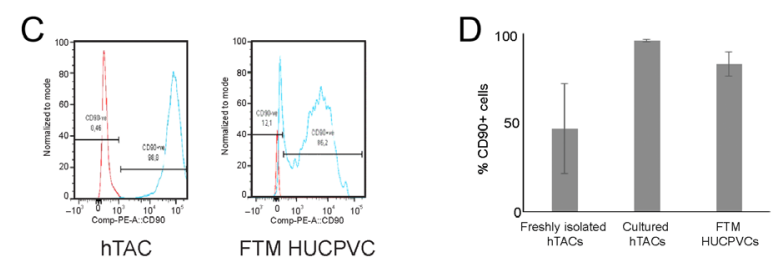

Figure 1 FTM HUCPVCs and human testicular adherent cells expanded in hSSC culture conditions (hTACs) have MSC-like phenotypes. (A) Representative bright field micrographs of hTACs and FTM HUCPVCs expanded for 2 passages in supplemented StemPro34-based culture conditions. Scale bar $=50 \mu \mathrm{m}$. (B and C) Representative micrographs of hTACs and FTM HUCPVCs immunostained for MSC-associated markers CD90 (red) and CD44 (green) (left panels); SOX9 (red) and CD44 (green) (middle panel) or where primary antibodies were omitted as negative controls (right panels), and counterstained with Hoechst (blue) to visualize nuclei. FTM HUCPVCs expanded in standard MSC conditions were used as positive controls. Scale bar $=20 \mu \mathrm{m}$. (D) Representative flow cytometry analysis plots for CD90 in hTACs (left panel) and FTM HUCPVCs (right panel). (E) Quantification of flow cytometric analysis of CD90+ve cells in freshly isolated hTACs, hTACs in culture for 2 passages and FTM HUCPVCs. 
A

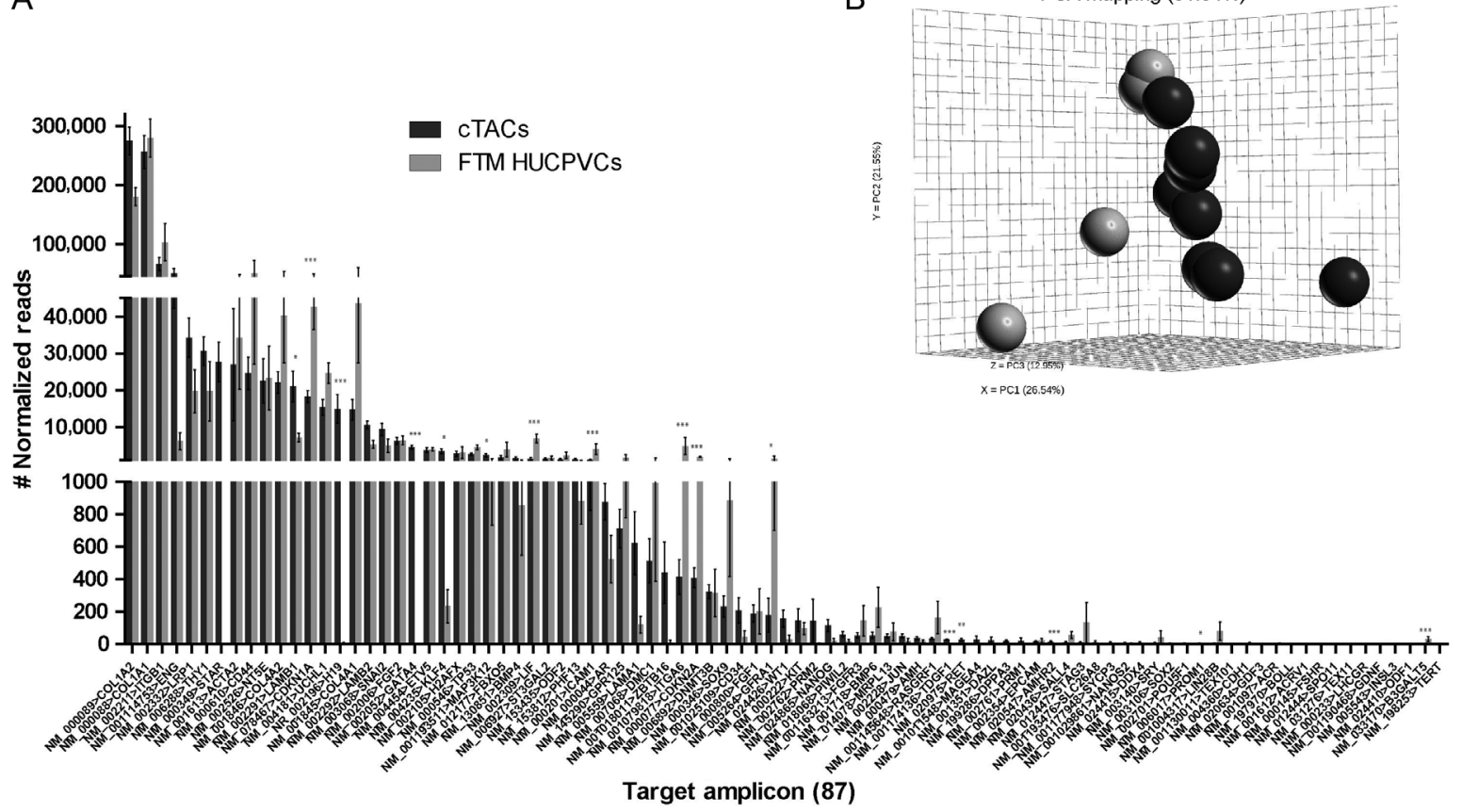

B

PCA mapping $(61.04 \%)$

C
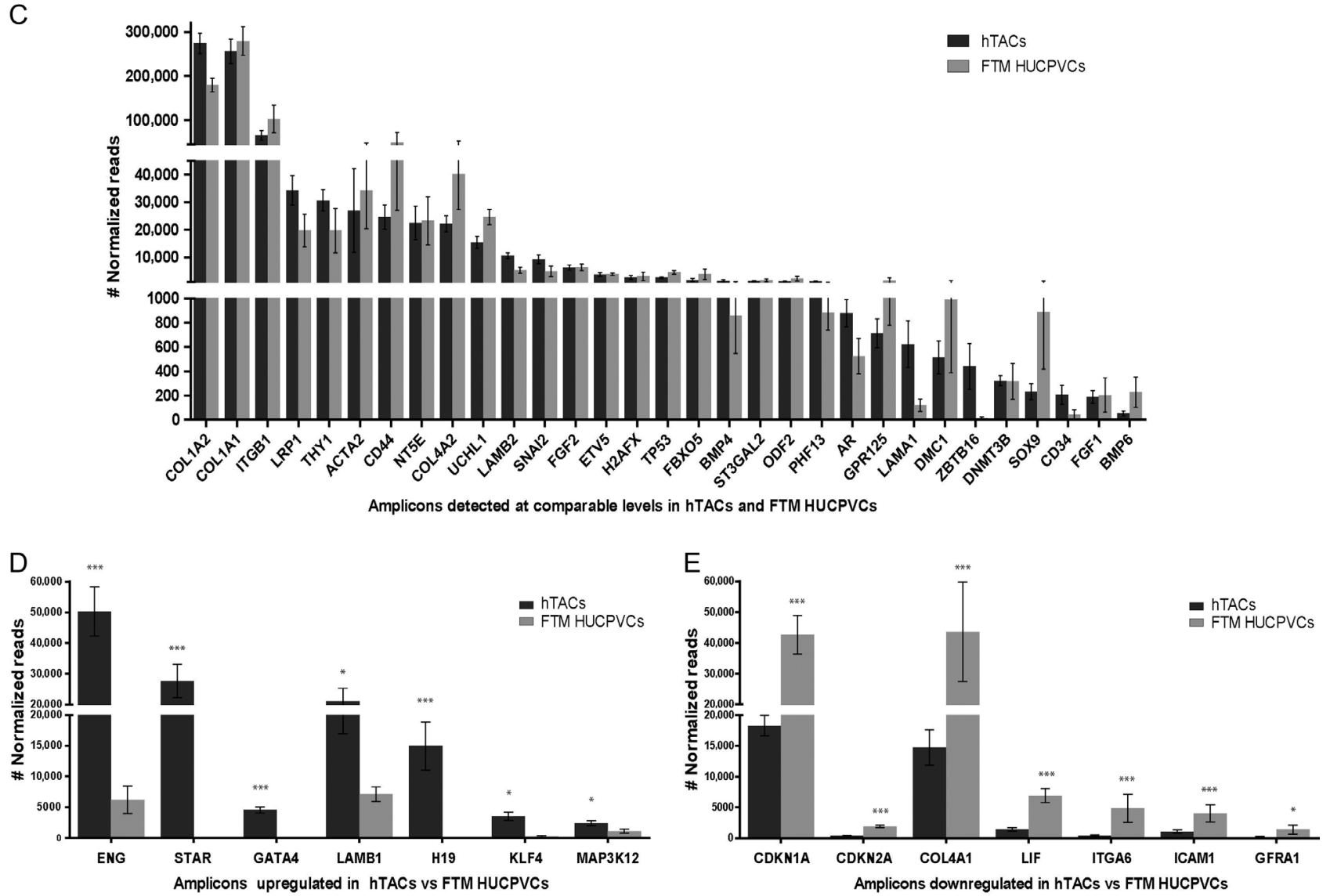

Figure 2 Targeted RNAseq profiling of CD90+ FTM HUCPVCs and hTACs. (A) Graphical representation of normalized number of reads for 87 targeted RNAseq amplicons in CD90+ hTACs (red) and CD90+ve FTM HUCPVCs (blue), sorted by highest to lowest gene expression in CD90+ hTACs. (B) Principle components analysis (PCA) map of 4 samples of hTACs (dark gray) and 8 samples of FTM HUPCVCs (light gray) represented in (A). (C, D and E) Graphical representations of highly expressed transcripts expressed at (C) similar levels, (D) significantly higher levels and (E) significantly lower levels in hTACs vs FTM HUCPVCs. ${ }^{*} P<0.05 ;{ }^{* *} P<0.01 ;{ }^{* *} P<0.005$. 

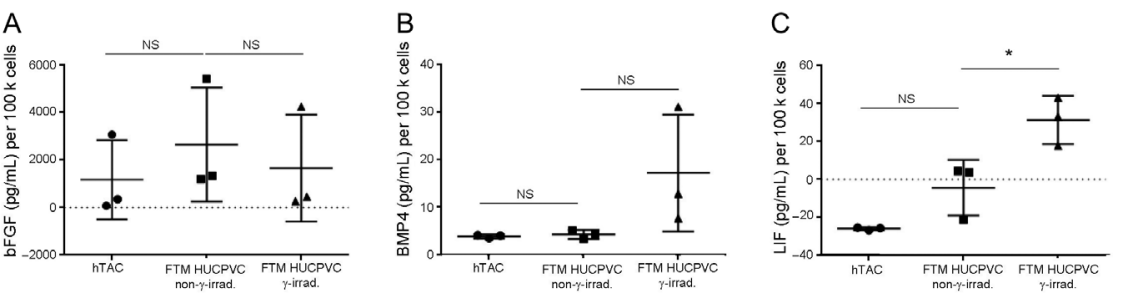

Figure 3 Growth factors in conditioned media of FTM HUCPVCs and hTACs. (A, B and C) Graphical representation of ELISA results for bFGF (A), BMP4 (B) and LIF (C) in hTACs, FTM HUCPVCs as well as gamma-irradiated $(\gamma$-irrad.) FTM HUCPVCs, where 3 independent samples of each cell type was analyzed. Each dot on the graph represents the mean of duplicate readings for each sample. GDNF and BMP6 were also assessed but were not detected (not shown). ${ }^{*} P<0.05$.
(Fig. 2D). Downregulated transcripts included the cell cycle-associated molecules CDKN1A and CDKN2A, testicular ECM-associated COL4A1, IL-6 family cytokine $L I F$, as well as cell adhesion molecules and growth factor receptors previously found to be associated with (but not specific to) the SSC phenotypes ITGA6, ICAM1 and GFRA1 $(P<0.05)$ (Fig. 2E).

\section{HUCPVCs express testicular extracellular matrix and niche-associated growth factors}

Both cell types also displayed relatively high levels of the extracellular matrix (ECM)-encoding transcripts COL1A2, COL1A1, COLAA2, LAMB1 and LAMB1 and relatively lower levels of $L A M A 1$. $L A M B 1$ was found to be differentially expressed between the cell types as discussed previously. Transcripts-encoding growth factors known to regulate SCCs and germ cell selfrenewal and homeostasis, including BMP4, FGF2 and $L I F$, were detected at high levels in both hTACs and FTM HUCPVCs (Fig. 2C), whereas FGF1, BMP6 and TDGF1 were detected at low levels. GDNF was not detected in either cell type. Only LIF was found to be differentially expressed (upregulated in FTM HUCPVCs). We assessed the levels of some of these secreted factors in the conditioned media of each cell type using ELISAs. bFGF and BMP4 were detected at comparable levels in hTAC and FTM HUCPVC conditioned media $(3.86 \pm 0.23 \mathrm{pg} /$ $\mathrm{mL}$ vs $4.29 \pm 0.53 \mathrm{pg} / \mathrm{mL},(P=0.69)$ and $1167.3 \pm 958.27$ vs 2652.20 \pm 1386.432 , $(P=0.10)$ respectively) (Fig. 3A and B). LIF was detected in 2 out of 3 established lines of FTM HUCPVCs but not in any of the hTAC samples (Fig. 3C). BMP6 and GDNF were not detected in the conditioned media of either cell type. Interestingly, gamma-irradiation treatment of FTM HUCPVCs significantly increased the secretion of LIF by these cells $(-4.32 \pm 8.46 \mathrm{pg} / \mathrm{mL}$ vs $31.39 \pm 12.72 \mathrm{pg} / \mathrm{mL}) \quad(P<0.05)$ (Fig. 3C). Although this treatment also appeared to increase BMP4 levels, this was not statistically significant $(P=0.3)$.

\section{FTM HUCPVCs support ex vivo expansion of murine germ cells including putative SSCs}

To determine whether FTM HUCPVCs could support germ cell survival and proliferation in vitro, we cultured murine germ cells on mitomycin-inactivated FTM HUCPVC feeders. We compared the ability of FTM HUCPVCs to support germ cell colony formation with that

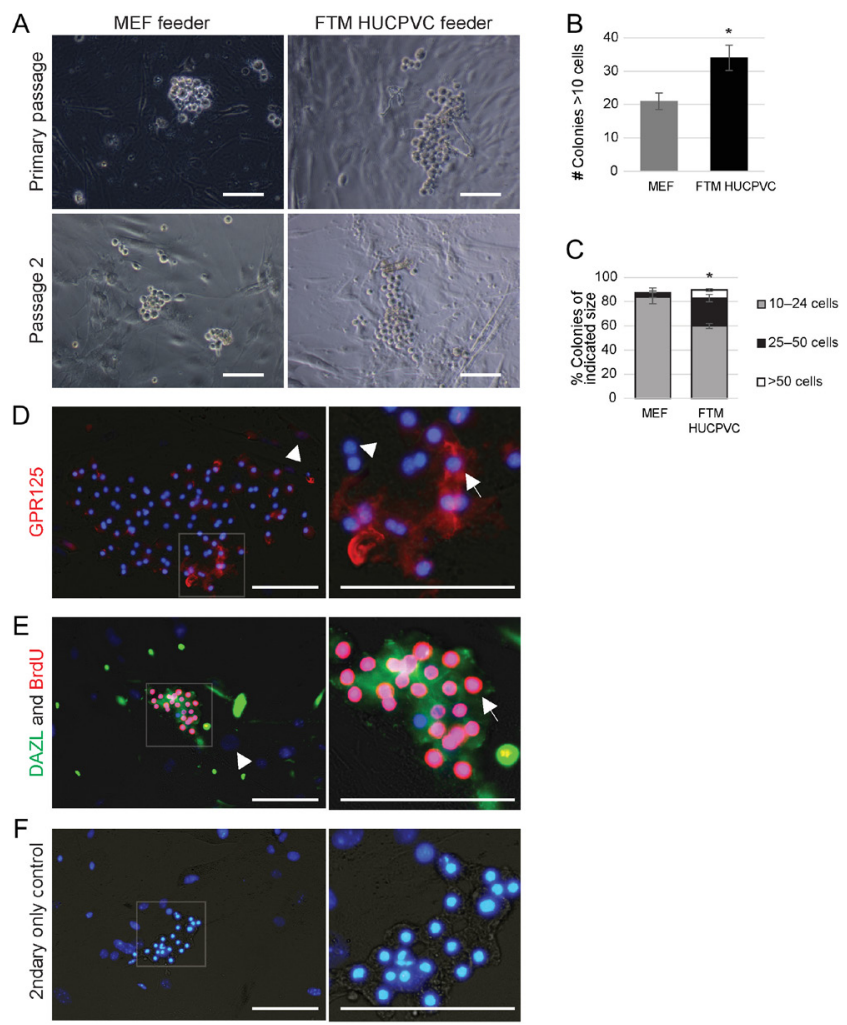

Figure 4 FTM HUCPVCs can support germ cell survival and self-renewal in vitro. (A) Micrograph of small grape-like putative mouse germ cell colony taken 14 days after unsorted mouse germ cells were plated on MEF (left) and FTM HUCPVC (right) feeders. (B and C) Quantification of * of colonies observed that were greater than 10 cells (B) and of colonies between 10 and 24 cells, 25 and 50 cells or larger than 50 cells $(C) . * P<0.05$. Micrograph of small grape-like putative mouse germ cell colony taken 14 days after mouse germ cells were plated on MEF (left) and FTM HUCPVC (right) feeders. Scale bar $=100 \mu \mathrm{m}$. (D, E and F) Micrographs of putative mouse germ cell colonies from FTM HUCPVC feeder cultures treated with BrdU and immunostained for the early germ cell marker GPR125 (D), the early germ cell marker DAZL and BrdU (E), secondary antibody only (F). Cells were counterstained with Hoechst (blue) to label nuclei. Bright field overlay is also shown. Scale bar $=100 \mu \mathrm{m}$. Left panels are $2.5 \times$ magnified of area demarcated by gray box in left panel. Arrows and arrowheads indicate immunopositive and immunonegative cells respectively. 
A

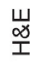

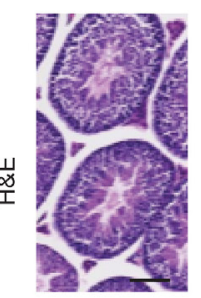

MEHP:

Treatment: Interval post-MEHP:
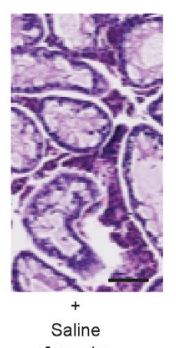
3 weeks

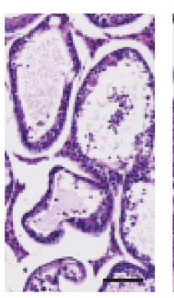

FTM HUCPVC 1 week

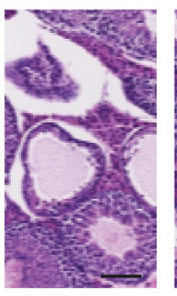

$\stackrel{+}{+}$ 2 weeks

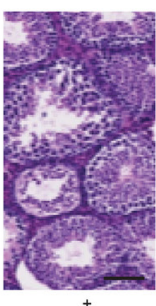

FTM HUCPVC
B

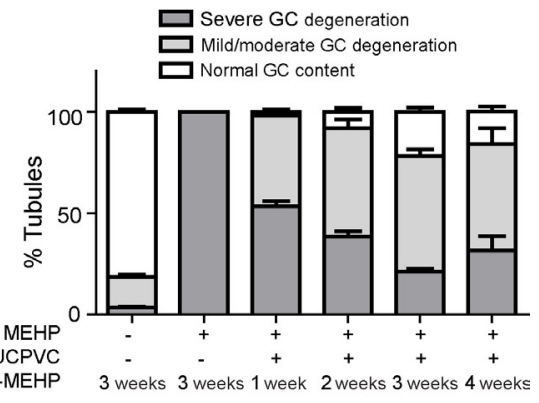

C

Corn oil + saline

$\mathrm{MEHP}+$ saline

MEHP + FTM HUCPVC

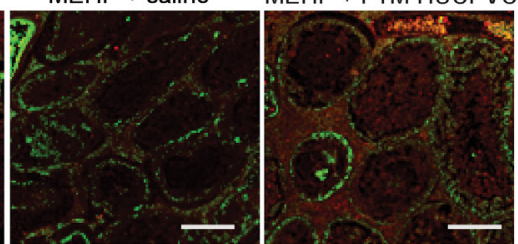

Negative control
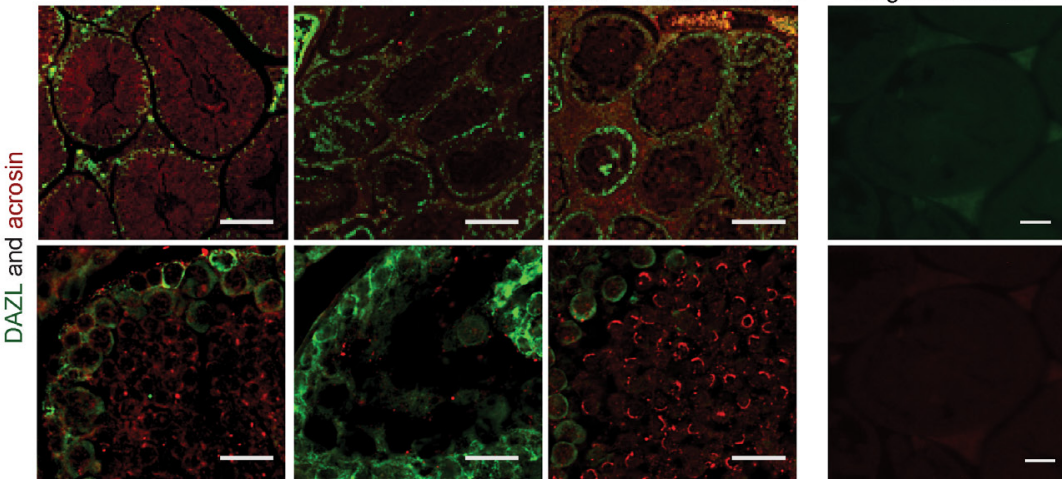

Figure 5 FTM HUCPVCs support germ cell regeneration in a model of MEHP-induced testicular injury. (A) H\&E staining of corn oil-treated control (left panel) and MEHP-treated mouse testis tissue section 3 weeks after intra-testicular injection with saline (2nd panel) and 1-3 weeks after intra-testicular injection with FTM HUCPVCs (panels 3-5). Scale bar $=100 \mu \mathrm{m}$. (B) Quantification of the percentage of tubules (out of more than 300 counted in at least 4 images) with (1) no damage (intact), with (2) aberrant number of germ cell layers but most germ cell (GC) layers present (aberrant cellular content) or (3) severe damage (completely destroyed). $n=2$ corn oil controls, $n=2 \mathrm{MEHP}+$ saline at 3 weeks, $n=1$ of each MEHP+FTM HUCPVC time points ${ }^{* * *} P<0.001$ saline vs MEHP at 3-4 weeks). (C) Represented low and high magnification micrographs of DAZL (green) and acrosin (red) fluorescence immunohistochemistry in each experimental group, at the 3-week time point. Scale bar $=100 \mu \mathrm{m}$ (top row); $14 \mu \mathrm{m}$ (bottom row). DAZL-positive cells are localized in outer whereas acrosin-positive (crescent) signal is mainly detected in inner germ cell layers respectively. Negative control micrograph represents corn oil + saline control tissue sections where primary antibodies were omitted (top panel, green channel; bottom panel, red channel). Scale bar $=100 \mu \mathrm{m}$.

of mouse embryonic fibroblast feeders (MEFs), which are often used as feeders to expand SSCs (Guo et al. 2014). Grape-like cell colonies, which have previously been reported to contain the SSCs (Kanatsu-Shinohara et al. 2003), were observed in germ cell cultures plated on MEF or FTM HUCPVC feeders within 10 days (Fig. 4A). A higher frequency of grape-like colonies containing greater than 10 cells were observed at the second passage in FTM HUCPVC feeder conditions Vs MEFs $(34 \pm 3.84$ vs $21 \pm 2.51$ respectively) $(P<0.05)$ (Fig. $4 \mathrm{~B})$. Furthermore, the proportion of these colonies containing greater than 25 cells was significantly increased in the FTM HUCPVC feeder conditions when compared to MEFs $(30 \pm 12 \%$ vs $4 \pm 3 \%, P<0.05)$ (Fig. 4C). Some experiments also included a BrdU incorporation assay at the second passage to determine whether colonies had formed by aggregation or germ cell proliferation. ICC confirmed that cells within these colonies (but not other somatic cells) were positive for GPR125 and DAZL expression, putative SSC-associated and early germ cellassociated markers respectively (Seandel et al. 2007,
Heetal.2010)(Fig.4D, EandF).Co-immunocytochemistry for these markers and BrdU showed that the majority of colonies contained cells that were immunopositive for both DAZL and BrdU (Fig. 4E) and GPR125 and BrdU (Supplementary Fig. 2). We were not able to expand germ cells beyond passage 2 in either condition. This is likely due to the proliferation of residual murine testicular adherent somatic cells (not fully removed after differential plating), which are known to overgrow and take over SSC cultures.

\section{FTM HUCPVCs support the regeneration of seminiferous tubules in a MEHP-induced Sertoli damage mouse model}

To determine whether the paracrine properties of FTM HUCPVCs could support testis niche regeneration, we performed localized injections of FTM HUCPVCs in an immunocompromised mouse model of mono2-ethylhexyl phthalate (MEHP)-induced testicular damage. We confirmed that, as previously published 
(Dalgaard et al. 2001), Sertoli cells are compromised by this treatment, leading to the obliteration of all late stages of spermatogenesis within $72 \mathrm{~h}$ (Supplementary Fig. 1A). PKH26-labeled FTM HUCPVCs or saline (control group) was injected $24 \mathrm{~h}$ after MEHP gavage. FTM HUCPVCs were found to localize mainly in the interstitial space, near the basal edges of seminiferous tubules (Supplementary Fig. 1B). We observed an incremental increase in the percentage of intact seminiferous tubules in testis tissue sections of animals that received an intra-testicular injection of FTM HUCPVCs from $2.0 \pm 0.1 \%$ at 1 week to up to $22 \pm 3.2 \%$ at 3 weeks after MEHP $(P<0.01)$. The increase was striking when compared to animals in the saline-treated control group, where we did not observe any intact tubules at 3 weeks after injury $(P<0.001)$ (Fig. 5A and C). At three weeks, intact seminiferous tubules from animals injected with FTM HUCPVCs after injury also showed grossly normal germ cell lineage structures, including DAZL+ve outer layers and acrosin+ve inner layers, whereas animals injected with saline (negative control) did not display such tubules (Fig. 5C).

\section{Discussion}

Our data support that FTM HUCPVCs mimic several key testicular niche characteristics and have supportive properties for testicular regeneration in vitro and in vivo. We demonstrated that FTM HUCPVCs have a strong phenotypical similarity to adherent testicular somatic cells expanded in culture, a cell type that has previously been shown to be required for ex vivo expansion of hSSCs (Smith et al. 2014). Both hTACs and FTM HUCPVCs demonstrated the expression of mesenchymal associated markers, extracellular matrix molecules and growth factors including FGF2, BMP4 and LIF that are known to support and regulate SSC maintenance, proliferation and differentiation in vivo. In addition, FTM HUCPVCs supported short-term murine germ cell colony expansion ex vivo when used as feeders and may even be superior to MEFs. Furthermore, our experiments suggest that FTM HUCPVCs possess testicular niche supportive properties in vivo, where they supported striking germ cell lineage regeneration compared to untreated controls in a mouse model of chemically induced seminiferous tubule degeneration.

The MSC properties of hTACs and FTM HUCPVCs expanded in hSSC culture conditions, including their immunophenotypical properties and ability to differentiate into mesenchymal cell lineages, have previously been demonstrated (Hong et al. 2013, Smith et al. 2014, respectively). The targeted RNAseq, immunocytochemistry/flow cytometry and ELISA data presented here expand upon and support previous studies demonstrating shared MSC-like properties between hTACs and FTM HUCPVCs. Although the perivascular region of the first trimester umbilical cord have been demonstrated to be rich in cells expressing MSC and pericyte protein markers (He et al. 2012, Hong et al. 2013), the origin of hTACs with MSC properties is not clear. Smith and coworkers proposed that these cells may originate in bone marrow. However, other reports (Crisan et al. 2008, Feng et al. 2011) have suggested that a subset of MSCs may have a perivascular (pericyte) origin and can be derived from vascularized tissues. Accordingly, hTACs with mesenchymal properties may be derived from the perivascular regions of microvessels in the interstitial testicular tissue. However, peritubular myoid cells express similar immunophenotypes, and based on the gene expression profile data presented here, cannot be excluded as an alternate source of hTACs. Taken together, our data suggest strong phenotypic similarities between hTACs and FTM HUCPVCs.

Our in vitro data support that FTM HUCPVCs have pro-survival and pro-mitotic effects on murine germ cells, including GPR125-expressing cells. These data suggest that FTM HUCPVCs could be used as feeders for the long-term expansion of hSSCs by replacing hTACs, which are available in limited quantities or which may be defective in tissue samples isolated from patients with absent or defective spermatogenesis (pre-pubertal and NOA samples respectively). We are currently testing this hypothesis using human spermatogonial stem cells. Our future studies will also determine whether term HUCPVCs, isolated at birth, have similar properties and could also be used in allogeneic cell therapy applications for fertility preservation. HUCPVCs are a rich source of accessible MSCs and are easily expanded in culture. As we have shown here, they mimic several key properties of human testicular somatic cells and are as good, if not better than, MEFs for expansion of murine germ cells.

The data presented here, and in our previous studies, also suggest that culture conditions regulate the paracrine properties of FTM HUCPVCs. This implies that FTM HUCPVCs are versatile and may exert different functions depending on their microenvironment. This study provides information to optimize pre-feeder/implantation culture conditions and supplementation requirements for use of FTM HUCPVCs in fertility preservation strategies. In this study where FTM HUCPVCs were grown for 2 passages in StemPro34-based hSSC ex vivo expansion conditions (as they would be as feeders), we did not detect the expression of GDNF as previously reported when these cells are expanded in standard MSC conditions (E Shlush, L Maghen, S Swanson, S Kenigsberg, S Moskovtsev, T A Barretto, A Gauthier-Fisher and C L Librach, unpublished observations). We also observed relatively lower levels of LIF and BMP4 in conditioned media when 
compared to previous studies that we performed with FTM HUCPVCs expanded in standard MSC conditions, altogether suggesting that pre-expansion of FTM HUCPVCs in standard MSC conditions may promote even greater supportive properties when compared to StemPro34-based conditions. Furthermore, gamma-irradiation, which prevents overgrowth of feeders, led to increased LIF levels in FTM HUCPVC cultures, an important factor for hSSC maintenance. It has yet to be determined whether co-culture with germ cells alters the paracrine profile of feeders. We hypothesize that the extracellular matrix and paracrine properties of hTACs and FTM HUCPVCs are important for their ability to support the testicular niche and propose future function-blocking experiments to confirm this.

Functional assays described here suggest that FTM HUCPVCs support germ cell regeneration after chemically induced testicular injury. Our results were similar to other studies in which bone marrow-derived MSCs were injected in rodent models of chemically induced testicular injury (Yang et al. 2014). The cellular mechanisms, however, remain to be fully elucidated. FTM HUCPVCs can be differentiated toward Sertoli-like and haploid germ-like cells when exposed to a stepwise cocktail of differentiation factors in vitro (Shlush et al. unpublished observations). We had initially hypothesized that, based on the possible mesenchymal origin of Sertoli cells (Wartenberg 1978, Byskov 1986), an alternate MSC source such as FTM HUCPVCs could replace some of the functions of Sertoli cells in animals treated with MEHP, a phthalate which targets Sertoli cells, leading to germ cell death. However, the in vitro data presented here suggest that undifferentiated FTM HUCPVCs, expanded in SSC culture conditions, which recapitulate the in vivo niche, did not differentiate into Sertoli-like or germ-like cells as they did not express high levels of Sertoli cell markers (WT1, FSHR, AMH and AMHR) or combinations of SSC or germ cell-specific markers. Furthermore, in this model, cells were injected directly into the testicular tissue, and not via the rete testis. We observed that the majority of FTM HUCPVCs were localized in the interstitial space. Therefore, it is unlikely that FTM HUCPVCs promoted germ cell regeneration by replacing Sertoli cells or directly contributing to the germ cell lineage, but likely promoted the regeneration of the germ cell lineages via paracrine mechanisms. We previously reported (Shlush et al. unpublished observations) the expression and secretion of testicular niche factors including FGF2, BMP4, LIF and GDNF in FTM HUCPVCs expanded in standard MSC growth conditions comprising aMEM $+10 \%$ FBS. We hypothesize that these and other paracrine properties of FTM HUCPVCs, injected within $24 \mathrm{~h}$ of MEHP administration, promoted the survival of Sertoli cells and SSCs after MEHP treatment, thereby leading to faster regeneration of the seminiferous tubule germ cell lineages when compared to control animals that received saline injections.

In conclusion, FTM HUCPVCs have the potential to be used as a novel standardized human feeder system for the xeno-free propagation of hSSCs and possibly in regenerative medicine applications related to fertility preservation. If successful, this strategy could have an important impact for fertility preservation on prepubertal boys undergoing gonadotoxic cancer therapy and/or for the treatment of infertile men with severely defective spermatogenesis, such as those with nonobstructive azoospermia.

\section{Supplementary data}

This is linked to the online version of the paper at http://dx.doi. org/10.1530/REP-16-0220.

\section{Declaration of interest}

Dr Clifford Librach has filed patent applications in Canada, U.S. and Australia for this invention entitled 'Method of Isolation and Use of Cells Derived from First Trimester Umbilical Cord Tissue'. The applications are currently pending and awaiting examination in Canada and U.S. but have received approval in Australia.

\section{Funding}

This work was funded by CReATe Program Inc.

\section{Author contribution statement}

L M contributed to study design, was involved in the conduction of all experiments, data collection and analysis and participated in co-wrote the manuscript. E S conceived the main ideas for the project, contributed to study design, data collection and analysis and edited the manuscript. I G contributed to experimental design and data collection related to human somatic cell characterization and revisions and edited the manuscript. M F performed some of the NGS and related data collection, confocal microscopy and edited the manuscript. T B co-performed the ELISAs, assisted with tissue culture and reviewed the manuscript. K J participated in sample collection, interpretation of results and edited the manuscript. K L participated in study design, sample collection, interpretation of results and edited the manuscript. A G F contributed to project conception, study design, performed data analysis, prepared figures and co-wrote the manuscript. $\mathrm{C} \mathrm{L}$ contributed to study design, interpretation of results and edited the manuscript.

\section{Acknowledgements}

The authors thank Kevin Quach (CReATe Fertility Centre) and Marie Deault-Bonin (Mount Sinai Hospital) for their assistance 
with the REB approval process, Saajida Bhorat, Marina Vainder and Junyan Li for their assistance with the data collection, as well as Paula Mackie, Shlomit Kenigsberg and Simon Alfred for assistance with NGS and bioinformatics. The authors thank Liran Shlush and Sasan Zandi (University Health Network) for their assistance with gamma-irradiation, and Denis Gallagher for his assistance with proof-reading the manuscript.

\section{References}

Byskov AG 1986 Differentiation of mammalian embryonic gonad. Physiological Reviews 66 71-117.

Choi NY, Park YS, Ryu JS, Lee HJ, Arauzo-Bravo MJ, Ko K, Han DW \& Scholer HR 2014 A novel feeder-free culture system for expansion of mouse spermatogonial stem cells. Molecular Cells 37 473-479. (doi:10.14348/molcells.2014.0080)

Crisan M, Huard J, Zheng B, Sun B, Yap S, Logar A, Giacobino JP, Casteilla L \& Peault B 2008 Purification and culture of human blood vessel-associated progenitor cells. Current Protocols in Stem Cell Biology Chapter 2 Unit 2B 2 1-2B 2 13. (doi:10.1002/9780470151808. sc02b02s4)

Dalgaard M, Hossaini A, Hougaard KS, Hass U \& Ladefoged O 2001 Developmental toxicity of toluene in male rats: effects on semen quality, testis morphology, and apoptotic neurodegeneration. Archives of Toxicology 75 103-109. (doi:10.1007/s002040000209)

Dominici M, Le Blanc K, Mueller I, Slaper-Cortenbach I, Marini F, Krause D, Deans R, Keating A, Prockop D \& Horwitz E 2006 Minimal criteria for defining multipotent mesenchymal stromal cells. The International Society for Cellular Therapy position statement. Cytotherapy 8 315-317. (doi:10.1080/14653240600855905)

Feng J, Mantesso A, De Bari C, Nishiyama A \& Sharpe PT 2011 Dual origin of mesenchymal stem cells contributing to organ growth and repair. PNAS 108 6503-6508. (doi:10.1073/pnas.1015449108)

Guo Y, Hai Y, Gong Y, Li Z \& He Z 2014 Characterization, isolation, and culture of mouse and human spermatogonial stem cells. Journal of Cellular Physiology 229 407-413. (doi:10.1002/jcp.24471)

He Z, Kokkinaki M, Jiang J, Dobrinski I \& Dym M 2010 Isolation, characterization, and culture of human spermatogonia. Biology of Reproduction 82 363-372. (doi:10.1095/biolreprod.109.078550)

He Z, Kokkinaki M, Jiang J, Zeng W, Dobrinski I \& Dym M 2012 Isolation of human male germ-line stem cells using enzymatic digestion and magnetic-activated cell sorting. Methods in Molecular Biology 825 45-57. (doi:10.1007/978-1-61779-436-0_4)

Hong SH, Maghen L, Kenigsberg S, Teichert AM, Rammeloo AW, Shlush E, Szaraz P, Pereira S, Lulat A, Xiao R et al. 2013 Ontogeny of human umbilical cord perivascular cells: molecular and fate potential changes during gestation. Stem Cells and Development 22 2425-2439. (doi:10.1089/scd.2012.0552)
Kanatsu-Shinohara M \& Shinohara T 2010 Germline modification using mouse spermatogonial stem cells. Methods in Enzymology 477 17-36. (doi:10.1016/s0076-6879(10)77002-6)

Kanatsu-Shinohara M, Ogonuki N, Inoue K, Miki H, Ogura A, Toyokuni S \& Shinohara T 2003 Long-term proliferation in culture and germline transmission of mouse male germline stem cells. Biology of Reproduction 69 612-616. (doi:10.1095/biolreprod.103.017012)

Oatley JM \& Brinster RL 2012 The germline stem cell niche unit in mammalian testes. Physiological Reviews 92 577-595. (doi:10.1152/ physrev.00025.2011)

Sadri-Ardekani H, Mizrak SC, van Daalen SK, Korver CM, RoepersGajadien HL, Koruji M, Hovingh S, de Reijke TM, de la Rosette JJ, van der Veen F et al. 2009 Propagation of human spermatogonial stem cells in vitro. JAMA 302 2127-2134. (doi:10.1001/jama.2009.1689)

Sadri-Ardekani H, Akhondi MA, van der Veen F, Repping S \& van Pelt AM 2011 In vitro propagation of human prepubertal spermatogonial stem cells. JAMA 305 2416-2418. (doi:10.1001/jama.2011.791)

Sarugaser R, Lickorish D, Baksh D, Hosseini MM \& Davies JE 2005 Human umbilical cord perivascular (HUCPV) cells: a source of mesenchymal progenitors. Stem Cells 23 220-229. (doi:10.1634/ stemcells.2004-0166)

Seandel M, James D, Shmelkov SV, Falciatori I, Kim J, Chavala S, Scherr DS, Zhang F, Torres R, Gale NW et al. 2007 Generation of functional multipotent adult stem cells from GPR125+ germline progenitors. Nature 449 346-350. (doi:10.1038/nature06129)

Smith JF, Yango P, Altman E, Choudhry S, Poelzl A, Zamah AM, Rosen M, Klatsky PC \& Tran ND 2014 Testicular niche required for human spermatogonial stem cell expansion. Stem Cells Translational Medicine $\mathbf{3}$ 1043-1054. (doi:10.5966/sctm.2014-0045)

Szaraz P, Librach M, Maghen L, Iqbal F, Barretto TA, Kenigsberg S, Gauthier-Fisher A \& Librach CL 2016 In vitro differentiation of first trimester human umbilical cord perivascular cells into contracting cardiomyocyte-likecells.Stem Cells Internationa/201613.(doi:10.1155/ 2016/7513252)

Wartenberg H 1978 Human testicular development and the role of the mesonephros in the origin of a dual Sertoli cell system. Andrologia 10 1-21. (doi:10.1111/j.1439-0272.1978.tb01306.x)

Yang RF, Liu TH, Zhao K \& Xiong CL 2014 Enhancement of mouse germ cell-associated genes expression by injection of human umbilical cord mesenchymal stem cells into the testis of chemical-induced azoospermic mice. Asian Journal of Andrology 16 698-704. (doi:10.4103/1008682X.129209)

Received 20 April 2016

First decision 30 May 2016

Revised manuscript received 20 October 2016

Accepted 25 October 2016 